\title{
Olsalazine is not superior to placebo in maintaining remission of inactive Crohn's colitis and ileocolitis: a double blind, parallel, randomised, multicentre study
}

\author{
N Mahmud, M A Kamm, J L Dupas, D P Jewell, C A O’Morain, D G Weir, D Kelleher
}

Department of Clinical Medicine, Trinity College, St James's Hospital, Dublin, Ireland

N Mahmud

D G Weir

D Kelleher

Department of Medicine and Gastroenterology, St Mark's Hospital, Northwick Park, UK M A Kamm

Department of Medicine and Gastroenterology, Hospital Nord, Amiens, France J L Dupas

Gastroenterology Unit, Radcliffe Infirmary, Oxford, UK

D P Jewell

Department of Clinical Medicine, Trinity

College, Meath and Adelaide Hospital, Tallaght, Dublin, Ireland

C A O'Morain

Correspondence to: Dr N Mahmud, Department of Clinical Medicine, Trinity College Dublin, Trinity Centre for Health Sciences, St James's Hospital, Dublin

8, Ireland.nmahmud@tcd.ie

Accepted for publication 29 January 2001

\begin{abstract}
Background and aims-The benefit of 5-aminosalicylic acid therapy for maintenance of remission in Crohn's disease is controversial. The primary aim of this study was to evaluate the prophylactic properties of olsalazine in comparison with placebo for maintenance of remission in quiescent Crohn's colitis and/or ileocolitis.

Methods-In this randomised, double blind, parallel group study of olsalazine versus placebo, 328 patients with quiescent Crohn's colitis and/or ileocolitis were recruited. Treatment consisted of olsalazine $2.0 \mathrm{~g}$ daily or placebo for 52 weeks. The primary end point of efficacy was relapse, as defined by the Crohn's disease activity index (CDAI) and by clinical relapse. Laboratory and clinical disease activity indicators were also measured. Safety analysis consisted of documentation of adverse events and laboratory values.

Results-No differences in the frequency of termination due to relapse or time to termination due to relapse were noted between the two treatment groups (olsalazine $48.5 \% v$ placebo $45 \%$ ) for either colitis or ileocolitis. The failure rate, defined as not completing the study, was significantly higher in olsalazine treated patients compared with placebo treated patients for the overall population (colitis and/or ileocolitis: olsalazine $65.4 \% \quad v \quad 53.9 \% ; \mathrm{p}=0.038)$. Similar failure rates were seen for patients with colitis. A significantly higher percentage of olsalazine treated patients experienced adverse gastrointestinal events. Drug attributed adverse events were reported more frequently in the olsalazine treated group with gastrointestinal symptoms being causally related to olsalazine treatment (olsalazine $40.7 \% v$ placebo $26.9 \%$; $=0.010)$. Back pain was reported significantly more often by the placebo treated group. However, serious medical events did not differ between the two groups. Adverse events led to more early withdrawals in the olsalazine treated group than in the placebo treated group; thus average time in the study for patients in the olsalazine treatment group was significantly shorter than that of patients in the placebo group. Conclusions-Patients treated with olsalazine were more likely to terminate
\end{abstract}

their participation in the trial than those taking placebo. This difference was not related to relapse of disease, as measured by CDAI and clinical measures, but rather was due to the development of intolerable adverse medical events of a non-serious nature related to the gastrointestinal tract. The gastrointestinal related events in the olsalazine treated group may be due to the difference in gastrointestinal status at baseline which favoured the placebo treatment group.

(Gut 2001;49:552-556)

Keywords: olsalazine; Crohn's disease; colitis; ileocolitis

Sulphasalazine has been in use for almost four decades as a therapeutic and prophylactic agent for the treatment of inflammatory bowel disease. The sulphapyridine moiety is now known to cause the majority of the idiosyncratic adverse reactions of sulphasalazine whereas 5-aminosalicylic acid (5-ASA) is the therapeutically active moiety. ${ }^{1-4}$ In recent years several sulphapyridine free 5-ASA products have been developed, such as olsalazine and mesalazine..$^{4-6}$

Olsalazine consists of two 5-ASA molecules linked by an azo bond. Like sulphasalazine, the azo bond of olsalazine is split in the colon, releasing both 5-ASA molecules to exert local therapeutic activity. ${ }^{67}$ Other formulations of oral 5-ASA use $\mathrm{pH}$ dependent coatings to release 5-ASA to the target tissue. ${ }^{78}$ Sulphasalazine has been shown to be superior to placebo in preventing recurrence in quiescent Crohn's disease. ${ }^{9}$ However, the role of mesalazine in the prevention of relapse in quiescent Crohn's disease is controversial according to published studies. ${ }^{10-12}$

Olsalazine is a well tolerated maintenance agent for the treatment of ulcerative colitis. ${ }^{13-15}$ The role of olsalazine in induction and maintenance of remission in Crohn's colitis and/or ileocolitis has not yet been evaluated.

This trial was designed to evaluate the prophylactic properties of olsalazine in comparison with placebo for maintenance of remission in quiescent Crohn's disease. This study was confined to patients with Crohn's colitis and/or ileocolitis. The trial was a randomised, double

Abbreviations used in this paper: ASA, 5-aminosalicylic acid; CDAI, Crohn's disease activity index; CRF, case report form. 
blind, parallel group study of 328 patients. Patients were treated with $2.0 \mathrm{~g}$ of olsalazine or $2.0 \mathrm{~g}$ of placebo daily for 52 weeks.

\section{Methods}

A total of 328 patients of either sex, aged 18 years or over with established Crohn's colitis and/or ileocolitis in complete remission were enrolled during February 1992 to January 1996 (demographic details are shown in table 1). Disease activity was monitored by the Crohn's disease activity index (CDAI) ${ }^{16}$ for one month prior to randomisation. Patients were treated with olsalazine or placebo for 52 weeks. CDAI measurements including haematocrit and routine blood biochemistry were performed at the pre-study visit ( -4 week), at the start of treatment ( 0 week), and at weeks 4,13 , 26,39 , and 52 .

\section{INCLUSION CRITERIA}

Patients aged 18 years or over with a diagnosis of Crohn's colitis and/or ileocolitis established radiologically and/or endoscopically and/or histologically within the five years before entry into study were included. All patients had a barium follow through/small bowel enema to assess the extent of small bowel disease. Patients in remission for at least one month prior to randomisation were included. Remission was defined clinically (as assessed by the investigator) and objectively by a CDAI score of less than 150. Written informed consent was obtained from all patients who participated in this study.

\section{EXCLUSION CRITERIA}

Patients were excluded from the study if they received steroid, azathioprine, or other immunosuppressive (other than 5-ASA) therapy one month prior to week -4 or during the study period. Other exclusion criteria included concomitant therapy with antibiotics for more than one month. Patients who were pregnant, intending to be pregnancy, or breast feeding, and those with clinically significant hepatic or renal insufficiency, strictures causing mechanical obstruction, fistulae, oral or symptomatic anal Crohn's disease, stoma or significant small bowel disease apart from terminal ileal disease, and patients with known hypersensitivity to salicylates were excluded.

Patients rejected at week 0 could be entered at a later stage if they met the inclusion criteria and none of the exclusion criteria. All patients entered but who were later found to have a CDAI $>150$ at visit 2 were withdrawn.

Data on all patients were recorded in case report forms (CRFs). An institutional review board responsible for assuring the rights and safety of research patients prospectively reviewed and approved the protocol.

\section{TREATMENT REGIMENS AND RANDOMISATION} PROCEDURE

Patients recruited to the study were assigned to either olsalazine or placebo treatment according to a computer generated randomisation. Randomisation was performed in blocks of consecutive patients within each centre.
Olsalazine was administered as $250 \mathrm{mg}$ capsules. The final maintenance dose of $2.0 \mathrm{~g}$ daily was to be established during a period of one month and was not changed thereafter. The full drug dose was achieved by successive dose escalation over an eight day period. Identical placebos were provided by Kabi Pharmacia.

\section{CONCOMITANT MEDICATION}

No other active medication for Crohn's disease was permitted. However, other medications, including antidiarrhoeals agents such as codeine and loperamide, were recorded on the CRFs.

EFFICACY ASSESSMENT AND PRIMARY END POINT The primary end point of efficacy was relapse, defined by a CDAI score $>150$ or an increase in the CDAI score by 60 or more from the baseline score at visit 2 (week 0 ). Clinical relapse was defined as the need for additional therapy or for surgery: in exceptional situations where CDAI criteria were not fulfilled.

\section{SAFETY ASSESSMENTS}

Safety assessments were carried out in all recruited patients at weeks $0,13,26$, and 52 and at the time of termination of the study. Parameters were routine haematology, biochemistry including serum urea/electrolyte, creatinine, bilirubin, aspartate transferase, and alkaline phosphatase, and urinalysis for protein and blood (sediment was measured if urine was positive for blood or protein).

\section{STATISTICAL ANALYSIS}

All analyses were produced using UNIX SAS version 6.09 . The sample size was initially calculated for per protocol analysis of relapse rate assuming a placebo relapse rate of 35\% (clinically important difference in relapse rate was defined as $15 \%$ ). Analyses were performed comparing the olsalazine treatment group with the placebo group. Unless otherwise specified, these analyses were conducted with a significance level of 0.05 and the sample size needed 138 evaluable patients per treatment group.

For demographic/baseline data, the analysis included only treatment in the model and was a comparison of the two treatment groups. Categorical data were analysed using the $\chi^{2}$ test. Continuous variables were analysed using one way ANOVA. The primary efficacy assessment was a comparison between treatment groups using $\chi^{2}$ tests of the frequency of termination due to relapse. In addition, time to termination due to relapse was evaluated using Kaplan-Meier curves and log rank tests. Adverse events were classified and coded using the World Health Organisation dictionary and were compared in the two treatment groups using Fisher's exact test. The protocol required that haematology and chemistry laboratory assays be compared at baseline (week 0) and change from baseline to weeks 13, 26, and 52 was analysed using the Kruskal-Wallis test. Changes within treatment groups were tested using Wilcoxon signed rank tests. 
Table 1 Demographic/quantitative variables

\begin{tabular}{lcc}
\hline Variable & Placebo $(n=160)$ & Olsalazine $(n=167)$ \\
\hline Age (y) & $38.4(1.1)$ & $40.0(1.1)$ \\
Weight (kg) & $68.8(1.2)$ & $69.0(1.1)$ \\
Age at first diagnosis of CD (y) & $31.9(1.1)$ & $32.9(1.0)$ \\
Time since first diagnosis of CD (y) & $6.56(0.59)$ & $7.18(0.54)$ \\
No months of continous clinical remission prior to treatment & $21.97(1.97)$ & $20.91(2.0)$ \\
CDAI total prior to treatment & $55.8(3.3)$ & $59.8(3.4)$ \\
No bowel movement/day prior to treatment & $1.9(0.1)$ & $2.1(0.1)$ \\
No liquid/soft stools in a week prior to treatment & $6.4(0.6)$ & $7.6(0.7)$ \\
\hline
\end{tabular}

Values are mean (SEM).

CDAI, Crohn's disease activity index.

Table 2 Qualitative baseline variables

\begin{tabular}{lcr}
\hline Variable & Placebo $(n=160)$ & Olsalazine $(n=167)$ \\
\hline Sex & & \\
$\quad$ Male & $80(50)$ & $70(41.9)$ \\
$\quad$ Female & $80(50)$ & $97(58.1)$ \\
Disease at ileocaecal junction & $79(49.4)$ & $89(53.3)$ \\
Disease in colon & $155(96.9)$ & $157(94.0)$ \\
$100 \%$ of large bowel remaining & $119(74.4)$ & $116(69.5)$ \\
$100 \%$ of small bowel remaining & $121(75.6)$ & $119(71.3)$ \\
Formed stools present & $86(53.8)$ & $71(42.8)$ \\
Concurrent illnesses & $41(25.6)$ & $34(20.4)$ \\
Bowel surgery performed & $43(26.9)$ & $59(35.3)$ \\
Current treatment to prevent relapse & $94(58.8)$ & $99(59.3)$ \\
\hline
\end{tabular}

Values are $\mathrm{n}(\%)$.

Table 3 Reasons for study termination

\begin{tabular}{lcc}
\hline Reason for study termination & Placebo $(n(\%))$ & Olsalazine $(n(\%))$ \\
\hline Completed study & $75(46.6)$ & $57(34.1)$ \\
Relapse (CDAI) & $42(26.1)$ & $40(24.0)$ \\
Clinical relapse & $17(10.6)$ & $15(9.0)$ \\
Serious adverse events & $1(0.6)$ & $2(1.2)$ \\
Intolerable adverse events & $10(6.2)$ & $33(19.8)$ \\
Disallowed concomitant medication & $2(1.2)$ & $4(2.4)$ \\
Patient consent withdrawn & $3(1.9)$ & $6(3.6)$ \\
Other protocol violation & $3(1.9)$ & $4(2.4)$ \\
Other & $2(1.2)$ & $1(0.6)$ \\
Unknown & $161(100)$ & $1(0.6)$ \\
Total reporting & &
\end{tabular}

CDAI, Crohn's disease activity index.

\section{Results}

DISPOSITION OF PATIENTS

A total of 328 patients (placebo $(n=161)$ and olsalazine $(n=167)$ ) were enrolled and randomised in this study. All patients except for one in the placebo group received the study drugs.

BASELINE DEMOGRAPHIC, QUANTITATIVE, AND QUALITATIVE VARIABLES

Seventy one of 167 (42.8\%) Crohn's disease patients in the olsalazine treated group reported having formed stools compared with 86 of $160(53.8 \%)$ patients in the placebo treated group $(\mathrm{p}=0.049)$. There were more women in the olsalazine treated group but the difference was not significant. All other variables were comparable between the two groups (tables 1, 2). Mean age of the patients was 39.2 (SEM 1.1) years. The diagnosis of Crohn's disease had been established in these patients for 6.87 (0.56) years and had been in continuous remission for about 21 months prior to treatment. Furthermore, the average CDAI score was 57.8 (3.3), and patients had on average two bowel movements a day and $7(0.65)$ liquid/soft stools in the week preceding the start of treatment. Ninety five per cent of patients enrolled in the study had colonic Crohn's disease and in $50 \%$ of these patients additional ileocaecal disease was present.
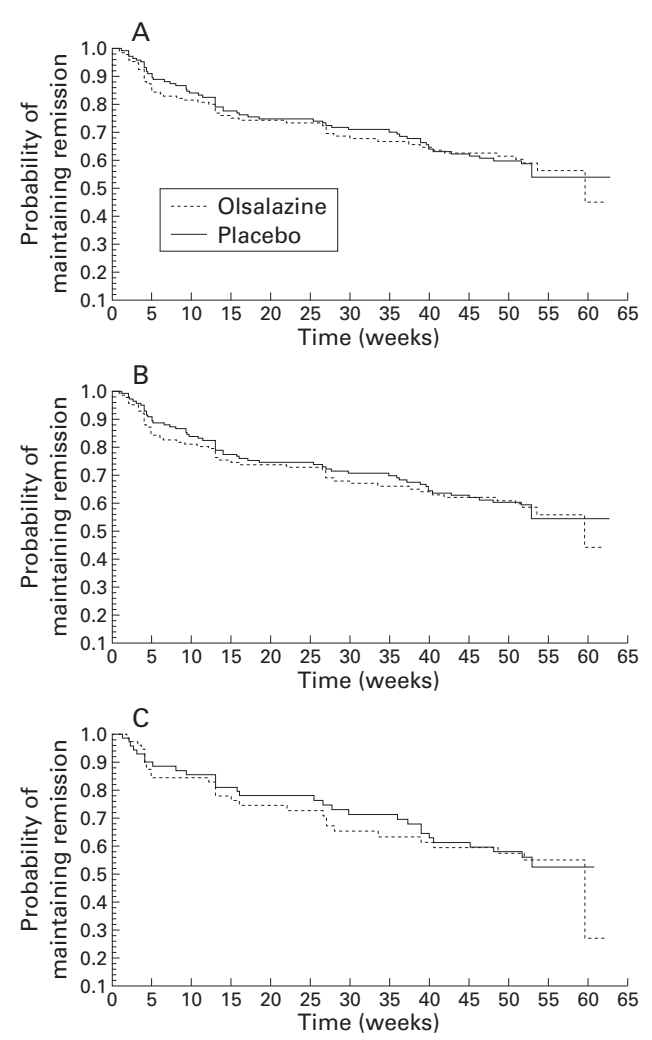

Figure 1 (A) Time to termination due to relapse in patients with Crohn's ileocolitis and/or colitis receiving maintenance therapy with olsalazine or placebo. (B) Time to termination due to relapse in patients with colonic Crohn's disease receiving maintenance therapy with olsalazine or placebo. (C) Time to termination due to relapse in patients with ileocaecal Crohn's disease receiving maintenance therapy with olsalazine or placebo.

REASONS FOR TERMINATION OF STUDY DRUG

The three main reasons for termination were relapse defined by CDAI (25\%), clinical relapse $(10 \%)$, and intolerable adverse events (13\%) (table 3). More patients in the olsalazine treated group failed to complete a 52 week treatment period than in the placebo treated group (olsalazine $65.9 \% v$ placebo $53.4 \%$ ). The relapse rate was similar in the two treated groups but the frequency of intolerable adverse events was higher in the olsalazine than in the placebo treated group $(19.8 \% v 6.2 \%$, respectively). Mean time in the study was significantly shorter for the olsalazine treated group compared with the placebo group (olsalazine 27.8 (1.66) $v$ placebo 34.6 (1.71) weeks; $\mathrm{p}=0.005)$.

\section{RELAPSE RATE AND TIME TO TERMINATION DUE} TO RELAPSE

The frequency of the relapse rate (monitored by CDAI and clinical relapse according to the site of disease - that is, colon and/or ileocaecal junction) was similar in the two treated groups (olsalazine $48.5 \% v$ placebo $45 \%$ ). Furthermore, no difference was observed in the two treatment groups when the time to termination due to relapse was analysed for disease at any site (fig 1).

TREATMENT FAILURE RATE

The overall failure rate was significantly higher in the olsalazine group compared with the placebo treated group in patients with colonic 
Table 4 Frequencies of drug attributed adverse events

\begin{tabular}{llll}
\hline & Placebo (n (\%)) & Olsalazine (n (\%)) & p Value \\
\hline Total No patients & 160 & 167 & \\
Patients with at least one related adverse event & $44(27.5)$ & $65(38.9)$ & 0.035 \\
WHO body system & & & \\
$\quad$ Gastrointestinal overall & $27(16.9)$ & $54(32.3)$ & 0.001 \\
$\quad$ Diarrhoea & $13(8.1)$ & $35(21.0)$ & 0.001 \\
$\quad$ Nausea & $3(1.9)$ & $11(6.6)$ & 0.053 \\
\hline
\end{tabular}

Crohn's and/or ileocaecal disease (olsalazine $65.4 \%$ v $53.9 \%$; $=0.038$ ). Furthermore, failure rate was also higher in the olsalazine treated compared with the placebo treated group in patients with ileocaecal disease (olsalazine $67.9 \%$ v placebo $54.8 \%$; $\mathrm{p}=0.095)$ and colonic disease alone $(65.4 \% v 53.6 \%$; $=0.035)$.

ADVERSE EVENTS

The majority of adverse events reported in the two treatment groups were mild to moderate in severity (table 4). The frequency of gastrointestinal related adverse events was significantly higher in patients treated with olsalazine compared with placebo (olsalazine $40.7 \%$ v placebo $26.9 \%$; $p=0.010)$. Diarrhoea frequency was $25.1 \%$ in the olsalazine treated group compared with $12.5 \%$ in the placebo group $(p=0.005)$. However, general body systems related adverse events such as backache were reported to be higher in the placebo group compared with the olsalazine treated group (placebo $3.1 \%$ v olsalazine $0 \%$; $=0.02$ ). There were no serious adverse events related to the study medication.

\section{Discussion}

There was no difference in the frequency of termination due to relapse or time to termination due to relapse between the two treatment groups for either colonic Crohn's disease or ileocolitis. Thus this study did not support the use of olsalazine (2 $\mathrm{g}$ /day) to maintain remission in patients with Crohn's colitis and/or ileocolitis. In randomised studies mesalazine has been shown to be superior to placebo in maintaining remission in inactive Crohn's disease with ileal disease. ${ }^{17}$

Florent et al studied endoscopic recurrences after curative resection for ileal, colonic, or ileocolonic Crohn's disease. ${ }^{18}$ Endoscopic relapse rates were $50 \%$ and $63 \%$ for the mesalazine (Claversal) and placebo groups, respectively. In this study there was a slight trend towards greater efficacy of mesalazine compared with placebo.

Thomson et al compared mesalazine (Mesasal/Claversal, 5-ASA) $1.5 \mathrm{~g}$ twice daily with placebo for maintenance of remission in 286 patients with Crohn's disease. ${ }^{19}$ The relapse rates of Crohn's disease were similar for up to 12 months in both the 5-ASA and placebo treatment groups. Furthermore, this study found no significant differences in those patients with Crohn's colitis or ileocolitis, or in those with ileitis with respect to relapse rates between the two treatment groups. ${ }^{19}$ Similar findings were noted in our study with the use of olsalazine $2.0 \mathrm{~g}$ /day compared with placebo; however, patients with ileal Crohn's disease alone were not studied.
An earlier study by Gendre et al demonstrated a significant benefit with the use of slow release mesalazine (Pentasa $2 \mathrm{~g}$ daily) in maintaining remission in Crohn's disease compared with placebo. ${ }^{20}$ The incidence of side effects was similar in both groups. Modigliani et al have also demonstrated the usefulness of mesalazine $4 \mathrm{~g}$ daily as maintenance therapy in steroid induced remission in Crohn's disease patients compared with placebo. ${ }^{21}$

More recently, in a multicentre placebo controlled study, $25 \%$ of patients with Crohn's disease maintained on mesalazine $4 \mathrm{~g}$ daily relapsed compared with $32 \%$ in the placebo treated group. ${ }^{22}$ A significantly reduced relapse rate was seen with mesalazine only in a subgroup of patients with isolated small bowel disease (mesalazine $22 \%$ v $40 \%$ placebo; $\mathrm{p}=0.02)$.

A recently published meta-analysis of 15 randomised controlled trials adjusted for confounding variables showed that mesalazine maintenance therapy significantly reduced the risk of symptomatic relapse of Crohn's disease. The benefit was mainly observed in the post-surgical setting, in patients with ileitis, and with prolonged disease duration. ${ }^{11}$ It is clear that olsalazine is not beneficial in Crohn's colitis and ileocolitis as evidence of side effects may be limiting. It is conceivable that gastrointestinal adverse events may be masking an occult beneficial effect on the disease process. However, based on the results of this study, olsalazine therapy could not be recommended for patients with Crohn's colitis/or ileocolitis.

In this study we have demonstrated that olsalazine at a dose of $2 \mathrm{~g} /$ day is not superior to placebo in maintaining remission of colonic or ileocolonic Crohn's disease. This is in contrast with the established therapeutic efficacy of this drug in ulcerative colitis. While 5-ASA derivatives have been shown to influence the course of Crohn's disease, the dose and nature of the delivery vehicle are important in determining outcome. The high rate of adverse events with olsalazine in the Crohn's disease cohort clearly limits potential efficacy in the maintenance of remission of this condition.

This study was supported by Kabi Pharmacia (now Pharmacia Upjohn). We gratefully acknowledge the assistance of Sven Ljungberg in performing the study. We also gratefully acknowledge the assistance of Valerie Trimble.

\section{Appendix}

OTHER MEMBERS OF THIS MULTICENTRE STUDY WERE AS FOLLOWS:

Republic of Ireland: J Crowe, J Lennon (Mater Hospital, Dublin); D O' Donoghue (St Vincent Hospital, Dublin); J Fielding (Beaumont Hospital, Dublin); Professor CF McCarthy, T O'Gorman (University College Hospital, Galway); Professor F Shanahan (University Hospital, Cork); JPD Long (Our Lady's Hospital, Drogheda, Co Louth). UK: TK Daneshmend (Royal Devon and Exeter Hospital, Exeter); Professor MJG Farthing (St Bartholomew's Hospital, London); IW Fellows (West Norwich Hospital, Norfolk); Professor CJ Hawkey (University Hospital, Queens Medical Centre, Nottingham); CD Holdsworth (Royal Hallamshire Hospital, Sheffield); V Mani (Leigh Infirmary, Lancashire); AP Manning (Bradford Royal Infirmary, West Yorkshire); DS Ramptom (Royal London Hospital, Whitechapel, London); ST Irwin, Professor TG Parks 
(Belfast City Hospital, Northern Ireland); RN Allan, RP Walt (The General Hospital, Steelhouse Lane, Birmingham); RPH Thompson (St Thomas' Hospital, London); F Murray (Ninewells Hospital, Dundee); KB Bardham (Rotherham Hospital, Rotherham); J Shaffer (Hope Hospital, Salford, Manchester); KR Palmer (Western General Hospital, Edinburgh); P McIntyre (Queen Elizabeth II Hospital, Herts). France: Professor Lemann (Hopital Saint-Louis, Paris); Professor Lerebours (Hopital Charles Nicolle, Rouen Cedex); Professor Colombel (CHR Hopital Claude Huriez, Lille Cedex).

1 Taffet SL, Das KM. Sulphasalazine. Adverse effects and desensitisation. Dig Dis Sci 1983;28:833-42.

2 Azad Khan AK, Piris J, Truelove SC. An experiment to determine the active therapeutic moiety of sulphasalazine. Lancet 1977:i :892-5.

3 van Hees PAM, Bakker JH, van Tongeren JHM. Effect of sulphapyridine, 5-aminosalicylic acid and placebo in patients with idiopathic proctitis: a study to determine the active therapeutic moiety of sulphaslazine. Gut 1980;21:632-5.

4 Myers B, Evan DNW, Rhode J, et al. Metabolism and urinary excretion of 5-aminosalicylic acid in healthy volunteers when given intravenously or released for absorption at different sites in gastrointestinal tract. Gut 1987;28:196-200.

5 Sandberg-Green II, Ryde M, Jarnerot G. Absorption and excretion of a single 1-g dose of asodiasal sodium in subjects with ileostomy. Scand $\mathcal{f}$ Gastroenterol 1983;18:107-11.

6 Willoughby CP, Aronsson JK, Agback H, et al. Distribution and metabolism in healthy volunteers of disodium and metabolism in healthy volunteers of disodium colitis. Gut 1982;23:1081-7.

7 Dew MJ, Hughes PJ, Lee MG, et al. An oral preparation to Dew MJ, Hughes PJ, Lee MG, et al. An oral preparation to
release drugs in the human colon. Br 7 Clin Pharmacol release drugs in

8 Dew MJ, Ryder REJ, Evan N, et al. Colonic release of 5-ASA from an oral preparation in active ulcerative colitis Br F Clin Pharmacol 1883;15:185-7.

9 Ewe K, Herfarth C, Malchow H, et al. Postoperative recurrence of Crohn's disease in relation to radicality of operation and sulphasalazine prophylaxis: A multicentre trial. Digestion 1989;42:224-32.

10 Thomson ABR, et al and the International Mesalazine Study Group. Coated oral 5-aminosalicylic acid versus placebo in maintaining remission of inactive Crohn's disease. Aliment Pharmacol Ther 1990;4:55-64.
11 Camma C, Giunta M, Rosselli M, et al. Mesalamine in the maintenance treatment of Crohn's disease: a meta-analysis
adjusted for confounding variables. Gastroenterology 1997; adjusted for conf

12 Sutherland LR, Martin F, Bailey RJ, et al. A randomized, placebo-controlled, double-blind trial of mesalamine in the maintenance of remission of Crohn's disease. The Canadian Mesalamine for Remission of Crohn's Disease Study Group. Gastroenterology 1997;112:1069-77.

13 Selby WS, Barr GD, Ireland A, et al. Olsalazine in active ulcerative colitis. BMf 1985;291:1373-5.

14 Sandberg-Gertzen $H$, Jarnerot G, Kraaz W. Azodisal sodium in the treatment of ulcerative colitis. A study of tolerance and relapse-prevention properties. Gastroenterology 1986; 90:1024-30.

15 Hertzwl J, Shearman DJC, Bochner F, et al. Azodisalicylate (olsalazine) in the treatment of ulcerative colitis. A placebo controlled clinical trial and assessment of drug disposition. f Gastroenterol Hepatol 1986;1:257-66.

16 Best WR, Becktel JM, Singleton JW, et al. Development of a Crohn's disease activity index. Nationdal Cooperative Crohn's Disease Study. Gastroenterology 1976;70:439-44.

17 Arber N, Odes HS, Fireman Z, et al. A controlled double blind multicenter study of effectiveness of 5-aminosalicylic acid in patients with Crohn's disease in remission. F Clin Gastroenterol 1995;20:203-6.

18 Florent C, Cortot A, Quandale P, et al. Placebo-controlled clinical trial of mesalazine in the prevention of early endoscopic recurrences after resection for Crohn's disease. Groupe d'Etudes Therapeutiques des Affections Inflammatoires Digestives (GETAID). Eur $\mathcal{F}$ Gastroenterol Hepatol 1996;8:229-33.

19 Thomson $\mathrm{AB}$, Wright JP, Vatn $\mathrm{M}$, et al. Mesalazine Mesasal/Claversal) $1.5 \mathrm{~g}$ b.d. vs. placebo in the maintenance of remission of patients with Crohn's disease. Aliment Pharmacol Ther 1995;9:673-83.

20 Gendre JP, Mary JY, Florent C, et al. Oral mesalamine (Pentasa) as maintenance treatment in Crohn's disease: a multicenter placebo controlled study. The Groupe d'Etudes Therapeutiques des Affections Inflammatoires Digestives. Gastroenterology 1993;104:435-9.

21 Modigliani R, Colombel JF, Dupas JL, et al. Mesalazine in Crohn's disease with steroid induced remission: effect on steroid withdrawal and remission maintenance, Groupe d'Etudes Therapeutiques des Affections Inflammatoires Digestives. Gastroenterology 1996;110:688-93.

22 Lochs H, Mayer M, Fleig WE, et al. Prophylaxis of postoperative relapse in Crohn's disease with mesalamine: European Cooperative Crohn's Disease Study VI. Gastroenterology 2000;118:264-73. 